\title{
Limitations of preserving volume in Allen-Cahn framework for microstructural analysis
}

\author{
P G Kubendran Amos ${ }^{\mathrm{a}, *}$, Ephraim Schoof ${ }^{\mathrm{a}}$, Jay Santoki ${ }^{\mathrm{a}, \mathrm{b}}$, Daniel Schneider ${ }^{\mathrm{a}, \mathrm{b}}$, Britta Nestler ${ }^{\mathrm{a}, \mathrm{b}}$ \\ ${ }^{a}$ Institute of Applied Materials (IAM-CMS), Karlsruhe Institute of Technology (KIT), \\ Strasse am Forum 7, 76131 Karlsruhe, Germany \\ ${ }^{b}$ Institute of Digital Materials Science (IDM), Karlsruhe University of Applied Sciences, \\ Moltkestr. 30, 76133 Karlsruhe, Germany
}

\begin{abstract}
Preserving volume in the Allen-Cahn framework is appealing as a computationally-efficient alternate for Cahn-Hilliard approach. The limitations of adopting volume-preserved Allen-Cahn treatment to analyse curvature-driven morphological transformations in chemical equilibrium is unraveled in the present work. The outcomes of redistribution-energy technique, which operates in Allen-Cahn framework, and a thermodynamically-consistent generalised quasi-AllenCahn (qAC) treatment, involving a conserved variable, is comparatively studied to explicate the limitations of the former. Analysis of representative microstructural evolution, in two- and threedimension, indicates that preserving volume in Allen-Cahn formalism renders an inaccurate transformation mechanism and final phase-distribution, which significantly deviate from the experimental observations and theoretical predictions. Moreover, it is shown that the redistributionenergy technique, in its existing form, fails to recover the thermodynamic condition imposed on the system.
\end{abstract}

Keywords: Spheroidization, morphological transformation, volume-preserve, microstructural stability, phase-field approach

\section{Introduction}

Applicability of a material, in a given environment, heavily relies on the stability of the microstructure under the associated thermodynamical condition [1]. The stability of a microstruc-

\footnotetext{
*Prince Gideon Kubendran Amos

Email address: prince.amos@kit.edu (P G Kubendran Amos)
} 
ture is either perturbed by phase transformation, or a change in the morphological configura5 tion of the constituent phases [2]. While the phase-changes alter the volume-fraction of the phases, during the morphological transformation, the respective phase-fractions are preserved all-through the evolution. In other words, the phase transformation is introduced by a deviation in the chemical equilibrium between the phases, in contrast, the shape-change occurs in the equilibrium governed by the curvature-difference in the morphology of the phases. The continuing equilibrium between the phases preserves the volume-fraction during the morphological evolution. Despite these differences, both phase and morphological transformation of a microstructure, significantly affect the properties of the material [3, 4]. Therefore, the stability of a microstructure, under a given thermodynamic condition, is exhaustively analysed to understand and predict the behaviour of a material.

Owing to the intricacies associated with the configuration and the evolution of a microstructure, theoretical treatments have always been adopted to complement and unravel the mechanisms undergirding the experimental observations [5, 6, 7]. In conventional techniques, contextually referred to sharp-interface approaches, the evolution of a microstructure is perceived by monitoring the temporal migration of the interface which separates the two phases. Tracking of the interface limits the applicability of this technique, despite its thermodynamical consistency. For instance, with increase in the morphological complexities of the evolving phases, the governing numerics become extremely convoluted [8, 9]. Moreover, singularity events like fragmentations cannot be directly formulated in sharp-interface approach. Therefore, phase-field technique is alternatively adopted to investigate microstructural evolutions which involve complex shapes and several singularity events.

Phase-field approach overcomes the limitations of the sharp-interface technique by circumventing the need for tracking the interface [10, 11]. This is achieved by introducing a scalar variable called phase-field $(\phi(\vec{x}, t))$, which is both spatially and temporally dependent. A section of a system wherein the phase-field assumes a constant value is referred to as a phase or bulk region. Correspondingly, region separating two phases, which is characterised by smoothly varying phase-field, is the interface. In the phase-field approach, as opposed to the conventional technique, the microstructural evolution reflects the spatio-temporal evolution of the scalar vari- 
able. Although the introduction of the phase-field replaces a conventional interface with a diffuse region, the overall thermodynamical consistency of the approach is verified by elaborate numer5 ical treatments [12, 12].

Based on phase-field the entire system is characteristically distinguished into interface and bulk region. Accordingly, the overall energy-density, as a Ginzburg-Landau functional, is expressed as

$$
\mathcal{F}(\phi(\vec{x}, t), \nabla \phi(\vec{x}, t), \eta(\vec{x}, t))=\mathcal{F}_{\text {intf }}(\phi(\vec{x}, t), \nabla \phi(\vec{x}, t))+\mathcal{F}_{\text {bulk }}(\eta(\vec{x}, t), \phi(\vec{x}, t)),
$$

where functionals $\mathcal{F}_{\text {intf }}(\phi(\vec{x}, t), \boldsymbol{\nabla} \phi(\vec{x}, t))$ and $\mathcal{F}_{\text {bulk }}(\eta(\vec{x}, t), \phi(\vec{x}, t))$ represent the contribution of the diffuse interface and bulk region, respectively. The driving-force for the phase transformation is introduced in the bulk contribution through an appropriate variable, $\eta(\vec{x}, t)[13,14]$. Depending on the system considered, the nature and the description of the variable changes [15, 16, 17].

For the microstructural evolution governed by the curvature-difference, and the ability of the system to reduce the interfacial-energy per unit volume, which is the primary focus on the present work, the contribution of the bulk region is deemed insignificant [18, 19]. Therefore, the overall energy-density, with only interface contribution, is written as

$$
\mathcal{F}(\phi(\vec{x}, t), \boldsymbol{\nabla} \phi(\vec{x}, t))=\underbrace{\int_{V}\left[\varepsilon \gamma|\boldsymbol{\nabla} \phi(\vec{x}, t)|^{2}+\frac{1}{\varepsilon} f_{\text {pen }}(\phi(\vec{x}, t))\right] \mathrm{d}}_{\mathcal{F}_{\text {intf }}(\phi(\vec{x}, t), \boldsymbol{\nabla} \phi(\vec{x}, t))} \vec{x},
$$

where the $V$ is the volume of the system. As indicated in the above Eqn. (2), the energy density exclusively formulated based on interface contribution comprises of two terms. The first term on the right side of Eqn. (2) is the gradient-energy density, where $\gamma$ and $\varepsilon$ are interfacial energy-density and length-scale prefactor that dictate the width of the diffuse interface [20]. The penalising potential, which ensures that constant value is assigned to the phase-field on the either ends of the diffuse interface, is the second term on the right side of Eqn. (2). Having appropriately defined the functional, the system is allowed to evolve towards a phenomenological decrease in the overall energy-density of the system.

The temporal evolution of the phase-field rendering curvature-driven transformations in a 
system exclusively defined by the interface contribution is formulated as

$$
\begin{aligned}
\tau \varepsilon \frac{\partial \phi(\vec{x}, t)}{\partial t} & =-\frac{\partial \mathcal{F}(\phi(\vec{x}, t), \boldsymbol{\nabla} \phi(\vec{x}, t))}{\partial \phi(\vec{x}, t)} \\
& =2 \varepsilon \gamma \Delta \phi(\vec{x}, t)-\frac{1}{\varepsilon} f_{\text {pen }}^{\prime}(\phi(\vec{x}, t)) .
\end{aligned}
$$

The microstructural changes corresponding to the above evolution equation ensures the progressive reduction in the overall energy-density. During the evolution of the phase-field, the relaxation of the constant $\tau$ maintains the stability of the interface [21]. Furthermore, in Eqn. (3), $\Delta \phi(\vec{x}, t)$ indicates the Laplacian of the phase-field, $\nabla^{2} \phi(\vec{x}, t)$, while the phase-field derivative of the penalising potential is represented by $f_{\text {pen }}^{\prime}(\phi(\vec{x}, t))$. The evolution Eqn. (3) is generally referred to as Allen-Cahn equation [22].

In order to estimate the kinetics of the evolution in the phase-field approach, a sharp contour is realised within the diffuse region. This contour, which is described as

$$
\Gamma=\{\vec{x} \in V \mid \phi(\vec{x}=0.5\}
$$

is monitored to estimate the transformation rate. In has been exhaustively reported that, when $\tau=1$, the evolution in the Allen-Cahn framework adheres to the relation

$$
v(\vec{x}, t)=K(\vec{x}, t), \quad \vec{x} \in \Gamma
$$

where $v(\vec{x}, t)$ and $K(\vec{x}, t)$ are the normal velocity and mean curvature, respectively [23, 24, 25]. In other words, the evolution of a phase, Eqn. (3), in the absence of a bulk driving-force is entirely dictated by the curvature.

Owing to the ability of the Allen-Cahn approach to recover the influence of the curvature on the evolution, this technique has been extensively employed in investigating curvature-driven energy-minimising transformation [26, 27, 26]. However, a characteristic feature associated with the evolution Eqn. (3) is that the phase-field, which denotes the volume fraction of a phase, is nonconserved. Consequently, the volume of the evolving phase is not preserved, but progressively changes with the transformation. Therefore, curvature-driven energy-minimising changes in a microstructure, which have been analysed through the Allen-Cahn technique, have largely been confined to grain growth [28, 29]. 
As introduced earlier, the curvature-driven morphological evolutions extend beyond grain growth. Particularly, under chemical-equilibrium, the shape-changes are characterised by the preservation of the phase-fractions [30, 31, 32]. Consequently, attempts have been made to preserve volume in the Allen-Cahn framework in order to extend its applicability. Preliminary treatments include an additional term in the Allen-Cahn evolution Eqn. (3), which thwarted any change in the volume-fraction during the transformation [33, 34, 35]. The evolution equation in its extended form reads

$$
\tau \varepsilon \frac{\partial \phi(\vec{x}, t)}{\partial t}=2 \varepsilon \gamma \Delta \phi(\vec{x}, t)-\frac{1}{\varepsilon} f_{\mathrm{pen}}^{\prime}(\phi(\vec{x}, t))+\lambda_{1}(t)
$$

where the term $\lambda_{1}(t)$ ensures that the volume of a phase at any given instant is equal to its initial volume,

$$
\int_{V} \phi(\vec{x}, t) \mathrm{d} \vec{x}=\int_{V} \phi^{o}(\vec{x}) \mathrm{d} \vec{x} \quad(:=\text { constant }) .
$$

The additional term $\lambda_{1}(t)$ in Eqn. (6), formulated as the Lagrange multiplier, is written as

$$
\lambda_{1}(t)=\frac{1}{\varepsilon} \int_{V} f_{\text {pen }}^{\prime}(\phi(\vec{x}, t)) \mathrm{d} \vec{x} .
$$

It is important to note that the term, that preserves the volume in the Allen-Cahn evolution Eqn. (6), $\lambda_{1}(t)$, exclusively exhibits temporal dependency while remaining independent of the spatial condition. Owing to this nature of the Lagrange multiplier, a non-local effect is introduced in the simulations, which becomes evident in phases with low fraction( [36] and references therein). In other words, the term $\lambda_{1}(t)$, preserved the volume of the phases only when they are above a critical limit. Therefore, the subsequent extension of this technique involved augmenting a prefactor, that rendered a spatio-temporal dependency to the volume-preserving term [37]. With the prefactor, the term in Eqn. (8) transforms to

$$
\lambda_{2}(\vec{x}, t)=\frac{\sqrt{2 f_{\text {pen }}(\phi(\vec{x}, t))}}{\varepsilon} \int_{V} f_{\text {pen }}^{\prime}(\phi(\vec{x}, t)) \mathrm{d} \vec{x} .
$$

The outcomes of the Allen-Cahn treatment, separately involving the terms in Eqns. (8) and (9), has been extensively compared to the conventional approach which preserves the volumefraction [38]. This technique that inherently preserves the phase-fraction by treating the phasefield as a conserved variable is generally referred to as Cahn-Hilliard approach [39]. The temporal 
evolution of the phase-field under the Cahn-Hilliard treatment is expressed as

$$
\varepsilon \frac{\partial \phi(\vec{x}, t)}{\partial t}=-\nabla \cdot\left[M(\phi(\vec{x}, t)) \boldsymbol{\nabla} \frac{\partial \mathcal{F}(\phi(\vec{x}, t), \nabla \phi(\vec{x}, t))}{\partial \phi(\vec{x}, t)}\right]
$$

where $M(\phi(\vec{x}, t))$ dictates the mobility of the diffuse interface [40]. The comparative analysis unraveled that the results of the volume-preserved Allen-Cahn and Cahn-Hilliard technique are significantly different. Despite the exhaustive investigation, the following critical aspects have not been addressed in this comparative study:

- A unique approach of preserving volume by introducing a redistribution-energy density as a bulk contribution [41, 42], as opposed to modifying the phase-field evolution equation, is not considered.

- The disparity in the morphological changes in relation to the microstructural transformation is not convincingly discussed.

Therefore, in the present work, volume-preserved morphological evolutions, which are pertinent to the observed microstructural transformations, rendered by the redistribution-energy based Allen-Cahn technique is analysed. Moreover, instead of the Cahn-Hilliard approach, the outcomes of the redistribution-energy treatment is compared to the results of a generalised 'quasiAllen-Cahn' (qAC) technique, wherein a conserved variable is introduced and solved to preserve the phase-fractions. The consistency of the qAC technique by separately verified by comparing it with the well-established Cahn-Hilliard approach.

\section{Phase-field models}

\subsection{Redistribution-energy technique}

The techniques, hitherto, adopted to preserve volume in the Allen-Cahn framework [33, 37], modify the phase-field evolution equation to preclude any change in the phase-fraction. In contrast, the redistribution-energy treatment averts any change in the volume of the evolving phases by augmenting a bulk-energy contribution [41, 42]. The functional that encompasses the overall 
energy-density of the system, for the redistribution-energy model, is expressed as

$$
\mathcal{F}(\phi(\vec{x}, t), \boldsymbol{\nabla} \phi(\vec{x}, t))=\int_{V}\left[\varepsilon \gamma|\nabla \phi(\vec{x}, t)|^{2}+\frac{1}{\varepsilon} f_{\text {pen }}(\phi(\vec{x}, t))+g(\phi(\vec{x}, t))\right] \mathrm{d} \vec{x},
$$

where $g(\phi(\vec{x}, t))$ represents the redistribution-energy density. In the present redistributionenergy approach, obstacle potential is employed to penalise the scalar variable [20, 43]. Correspondingly, the penalising energy-density is expressed as

$$
\frac{1}{\varepsilon} f_{\mathrm{pen}}(\phi(\vec{x}, t))= \begin{cases}\underbrace{\frac{\gamma}{\varepsilon} \frac{16}{\pi^{2}} \phi(\vec{x}, t)[1-\phi(\vec{x}, t)]}_{\equiv \frac{1}{\varepsilon} f_{\mathrm{ob}}(\phi(\vec{x}, t))}, & \phi \in\{\mathcal{G} \equiv[0,1]\} \\ \infty & \phi \in\{\mathcal{G} \equiv[0,1]\} .\end{cases}
$$

In Eqn. (12), the Gibbs simplex which heavily penalises the phase-field when it is not bound by $[0,1]$ is represented by $\mathcal{G}$.

For the current two-phase consideration, the redistribution energy-density is written as the interpolation of the individual contributions, $\chi^{\alpha}(t)$ and $\chi^{\beta}(t)$,

$$
g(\phi(\vec{x}, t))=\chi^{\alpha}(t) h(\phi(\vec{x}, t))+\chi^{\beta}(t)[1-h(\phi(\vec{x}, t))],
$$

where $h(\phi(\vec{x}, t))$ is the interpolation function of the form $h(\phi)=\phi^{2}(3-2 \phi)$. The formulation of the redistribution-energy density in Eqn. (13) inherently circumvents any non-local effects in the numerical treatment.

The temporal evolution of the phase-field in the redistribution-energy framework is expressed as

$$
\tau \varepsilon \frac{\partial \phi(\vec{x}, t)}{\partial t}=2 \varepsilon \gamma \Delta \phi(\vec{x}, t)-\frac{\gamma}{\varepsilon} \frac{16}{\pi^{2}}[1-2 \phi(\vec{x}, t)]-g^{\prime}(\phi(\vec{x}, t))
$$

where $g^{\prime}(\phi(\vec{x}, t))$ is the first derivative of the redistribution energy-density that reads

$$
g^{\prime}(\phi(\vec{x}, t))=\left[\chi^{\alpha}(t)-\chi^{\beta}(t)\right] \frac{\partial h(\phi(\vec{x}, t))}{\partial \phi(\vec{x}, t)} .
$$

The phase-field derivative of the redistribution-energy density, in Eqn. (15), acts as the driving force for preserving the phase-fraction during the evolution. 
The redistribution-energy technique, in principle, operates by allowing the interface to evolve locally under the influence of the curvature. However, when the evolution is accompanied by a decrease in the volume, the loss is compensated through the driving force $g^{\prime}(\phi(\vec{x}, t))$, which introduces an appropriate local interface-migration elsewhere, thereby counterbalancing any change in the phase-fractions.

\subsection{Generalised quasi-Allen-Cahn approach}

An alternate approach for preserving volume can be motivated from multiphase physical systems, wherein each phases are characterised by definite features like density or concentration. For instance, in a pure (or single-component) system, at the triple-point of its unary phasediagram, three phases which are distinguished by their densities co-exist in a definite proportion. Any change in this phase-fraction is solely established by an appropriate phase transformation that demands a driving-force. Therefore, by associating the phases with an additional scalarvariable, and imposing an equilibrium through this newly introduced parameter, precludes any phase transformation, thereby ensuring temporally unchanging volume [44, 45]. While the additional variable, which is generally conserved, aides in preserving the phase-fraction, the ability of the system to reduce the interfacial energy actuates the curvature-driven transformation. Similar approach has already been adopted in previous works by employing concentration to establish the volume-preserving equilibrium [46, 47]. However, it is conceivable that such physical attributions often limits the applicability of the treatment. Accordingly, in the present analysis, a phase-field model referred to as generalised quasi-Allen-Cahn (qAC) technique is formulated based on a non-specific conserved variable, $\eta$.

Reflecting its motivation, and akin to the redistribution technique delineated in the previous section, the volume fraction of the phases in the generalised $\mathrm{qAC}$ treatment is preserved by the bulk contribution. Correspondingly, the overall energy-density of the system with the newly introduced conserved variable is expressed as

$$
\mathcal{F}\left(\phi(\vec{x}, t), \nabla \phi(\vec{x}, t), \eta^{\alpha}(\vec{x}, t), \eta^{\beta}(\vec{x}, t)\right)=\int_{V}\left[\varepsilon \gamma|\nabla \phi(\vec{x}, t)|^{2}+\frac{1}{\varepsilon} f_{\mathrm{ob}}(\phi(\vec{x}, t))+f_{\mathrm{vp}}\left(\eta^{\alpha}, \eta^{\beta}, \phi\right)\right] \mathrm{d} \vec{x},
$$


where $f_{\mathrm{vp}}\left(\eta^{\alpha}, \eta^{\beta}, \phi\right)$ represents the energy density of the phases which facilitates volume preservation. Since treating $\eta$ as a continuous variable lend itself to a non-physical coupling of the interface and bulk contributions, phase dependent variables, $\eta^{\alpha}$ and $\eta^{\beta}$, are considered in the above formulation. The overall volume preserving energy-density in Eqn. 16), $f_{\mathrm{vp}}\left(\eta^{\alpha}, \eta^{\beta}, \phi\right)$, is expressed as the interpolation of individual phase contributions,

$$
f_{\mathrm{vp}}\left(\eta^{\alpha}(\vec{x}, t), \eta^{\beta}(\vec{x}, t), \phi(\vec{x}, t)\right)=f_{\mathrm{vp}}^{\alpha}\left(\eta^{\alpha}\right) h(\phi)+f_{\mathrm{vp}}^{\beta}\left(\eta^{\beta}\right)(1-h(\phi))
$$

In above Eqn. (17), the function which interpolates the individual volume-preserving energydensities, $f_{\mathrm{vp}}^{\alpha}$ and $f_{\mathrm{vp}}^{\beta}$ is identical to the one adopted in the redistribution-energy formalism (Eqn. (13)).

In order to establish an equilibrium, which ensures volume preservation, by the appropriate initialisation of the system through the newly introduced variables, $\eta^{\alpha}$ and $\eta^{\beta}$, the energy contributions of the individual phases are formulated as

$$
\begin{aligned}
& f_{\mathrm{vp}}^{\alpha}\left(\eta^{\alpha}(\vec{x}, t)\right)=A^{\alpha}\left[\eta^{\alpha}\right]^{2}+B^{\alpha} \eta^{\alpha}+D^{\alpha} \\
& f_{\mathrm{vp}}^{\beta}\left(\eta^{\beta}(\vec{x}, t)\right)=A^{\beta}\left[\eta^{\beta}\right]^{2}+B^{\beta} \eta^{\beta}+D^{\beta},
\end{aligned}
$$

where $A^{\Theta}, B^{\Theta}$ and $D^{\Theta}$, with $\Theta \in\{\alpha, \beta\}$, are parabolic coefficients. The description of individual contributions in Eqn. (18), $f_{\mathrm{vp}}^{\alpha}$ and $f_{\mathrm{vp}}^{\beta}$, is partially motivated by the geometrical nature of the freeenergy densities of the phases in binary systems. This polynomial formulation of the individual energy-densities uniquely characterises the volume-preserving equilibrium. In other words, the first derivatives of the individual phase-contributions are equal,

$$
\frac{\partial f_{\mathrm{vp}}^{\alpha}\left(\eta^{\alpha}\right)}{\partial \eta^{\alpha}}=\frac{\partial f_{\mathrm{vp}}^{\beta}\left(\eta^{\beta}\right)}{\partial \eta^{\beta}} \equiv \mu_{\mathrm{vp}}^{\alpha}=\mu_{\mathrm{vp}}^{\beta}=\mu_{\mathrm{vp}},
$$

when the interacting phases $\alpha$ and $\beta$ are in equilibrium. In Eqn. (19), $\mu_{\mathrm{vp}}$ denotes the volumepreserving potential. Furthermore, the description of the individual bulk-contributions in Eqn. (18) favours the invertibility of the phase-dependent variable, $\eta^{\alpha}$ and $\eta^{\beta}$, and the corresponding volume-preserving potential, $\mu_{\mathrm{vp}}^{\alpha}$ and $\mu_{\mathrm{vp}}^{\beta}$. Considering the phases are in equilibrium across the interface, the invertibility allows the treatment of the volume-preserving potential as the continuous variable, and ascertaining the spatially-varying phase-dependent variables. 
A homogenised parameter, expressed as

$$
\eta\left(\eta^{\alpha}, \eta^{\beta}, \phi(\vec{x}, t)\right)=\eta^{\alpha} h(\phi)+\eta^{\beta}[1-h(\phi)]
$$

the diffuse interface. A smooth-monotonic function relating individual phase-contributions in Eqn. (17) is adopted to interpolate $\eta^{\alpha}$ and $\eta^{\beta}$.

The temporal evolution of phase-field for the present formulation of the generalised qAC approach is expressed as

$$
\tau \varepsilon \frac{\partial \phi(\vec{x}, t)}{\partial t}=2 \varepsilon \gamma \Delta \phi(\vec{x}, t)-\frac{\gamma}{\varepsilon} \frac{16}{\pi^{2}}[1-2 \phi(\vec{x}, t)]-\frac{\partial f_{\mathrm{vp}}(\eta, \phi)}{\partial \phi},
$$

where $\tau$ is the relaxation constant. The driving-force for the phase-field evolution is dictate by $\frac{\partial f_{\mathrm{vp}}(\eta, \phi)}{\partial \phi}$, which is estimated as

$$
\frac{\partial f_{\mathrm{vp}}(\eta, \phi)}{\partial \phi}=\left\{f_{\mathrm{vp}}^{\alpha}(\eta)-f_{\mathrm{vp}}^{\beta}(\eta)-\mu_{\mathrm{vp}}\left[\eta^{\alpha}(\vec{x}, t)-\eta^{\beta}(\vec{x}, t)\right]\right\} \frac{\partial h(\phi)}{\partial \phi}
$$

owing to the consideration of phase-dependent variables, and their treatment across the interface, Eqn. (19).

Eqns. (21) and (22) indicate that the evolution of phase-field is primarily governed by the volume-preserving potential, $\mu_{\mathrm{vp}}$. When a two-phase system is initialised by assigning minimas of the parabolic energy-densities of the individual phases, an equilibrium which counteracts any phase transformation is established. This equilibrium consequently renders an equal volumepreserving potential across the interface. However, the introduction of a curvature-difference, effects the potential in accordance with the Gibbs-Thomson relation [48, 49]. The deviation from the equilibrated $\mu_{\mathrm{vp}}$ induces a definite driving-force ultimately resulting in a phase-field evolution governed by the curvature. In other words, while the appropriate initialisation of the system through the phase-dependent variables, $\eta^{\alpha}$ and $\eta^{\beta}$, establishes an equilibrium that preserves volume, the influence of curvature on the potential actuates the morphological transformation.

Owing to the dominant role of the volume-preserving potential, $\mu_{\mathrm{vp}}$, in directing the morphological changes, its temporal evolution is solved instead of the conserved variables. However, the 
evolution of the potential is formulated by considering the changes in the homogenised variable, $\eta$, with time. Based on Eqns. (19) and 20), the temporal evolution of the $\eta$ can be expressed as

$$
\frac{\partial \eta\left(\eta^{\alpha}, \eta^{\beta}, \phi\right)}{\partial t}=\frac{\partial \eta}{\partial \phi} \frac{\partial \phi}{\partial t}+\frac{\partial \eta}{\partial \mu_{\mathrm{vp}}} \frac{\partial \mu_{\mathrm{vp}}}{\partial t} .
$$

Moreover, the evolution of this homogenised variable in response to the gradient in volumepreserving potential induced by the curvature-difference is independently written as

$$
\frac{\partial \eta(\vec{x}, t)}{\partial t}=\boldsymbol{\nabla} \cdot\left[M(\phi) \nabla \mu_{\mathrm{vp}}(K)\right]
$$

where $K$ is the mean curvature. Since the $\eta$ is a conserved variable, its evolution equation adopts a form like Cahn-Hilliard relation. The parameter $M(\phi)$ in Eqn. (24) is mobility, which dictates the evolution rate of the homogenised variable, $\eta$. Different forms of mobility, including a scalar constant, can be adopted to effect the kinetics of $\eta$ evolution. However, in the current framework, a rather sophisticated formulation which includes the geometrical feature of the individual energy-contributions in adopted. The mobility involved in Eqn. (24) reads

$$
M(\phi)=D^{\alpha}\left(\frac{\partial^{2} f_{\mathrm{vp}}^{\alpha}}{\partial \eta^{2}}\right)^{-1} h(\phi)+D^{\beta}\left(\frac{\partial^{2} f_{\mathrm{vp}}^{\beta}}{\partial \eta^{2}}\right)^{-1}(1-h(\phi)),
$$

where $D^{\alpha}$ and $D^{\theta}$ are constants which relate to the diffusion coefficients in each phases, and can be suitably chosen to depict the nature of the bulk-phases. Furthermore, given the description of the individual phase-contribution in Eqn. (18), the terms $\frac{\partial^{2} f_{\mathrm{vp}}^{\alpha}}{\partial \eta^{2}}$ and $\frac{\partial^{2} f_{\mathrm{vp}}^{\beta}}{\partial \eta^{2}}$ are equivalent to the parabolic coefficients, $2 A^{\alpha}$ and $2 A^{\beta}$, respectively. By combining Eqns. (23) and (24), the temporal evolution of the potential, which primarily governs the volume-preserved curvature-driven transformations, is written as

$$
\frac{\partial \mu_{\mathrm{vp}}}{\partial t}=\left\{\boldsymbol{\nabla} \cdot\left[M(\phi) \nabla \mu_{\mathrm{vp}}(K)\right]-\left(\eta^{\alpha}-\eta^{\beta}\right) \frac{\partial h(\phi)}{\partial \phi}\right\}\left\{\frac{\partial \eta}{\partial \mu_{\mathrm{vp}}}\right\}^{-1}
$$

where, owing to the invertibility of the individual energy-densities, $\left\{\frac{\partial \eta}{\partial \mu_{\mathrm{vp}}}\right\}^{-1}$ is the inverse of the second derivative of matrix contribution $\left(\frac{1}{2 A^{\beta}}\right)$.

\section{Results and discussion}

In the present work, the limitations of preserving volume in the Allen-Cahn framework is explicated by comparing the outcomes of the redistribution-energy technique with that of the 


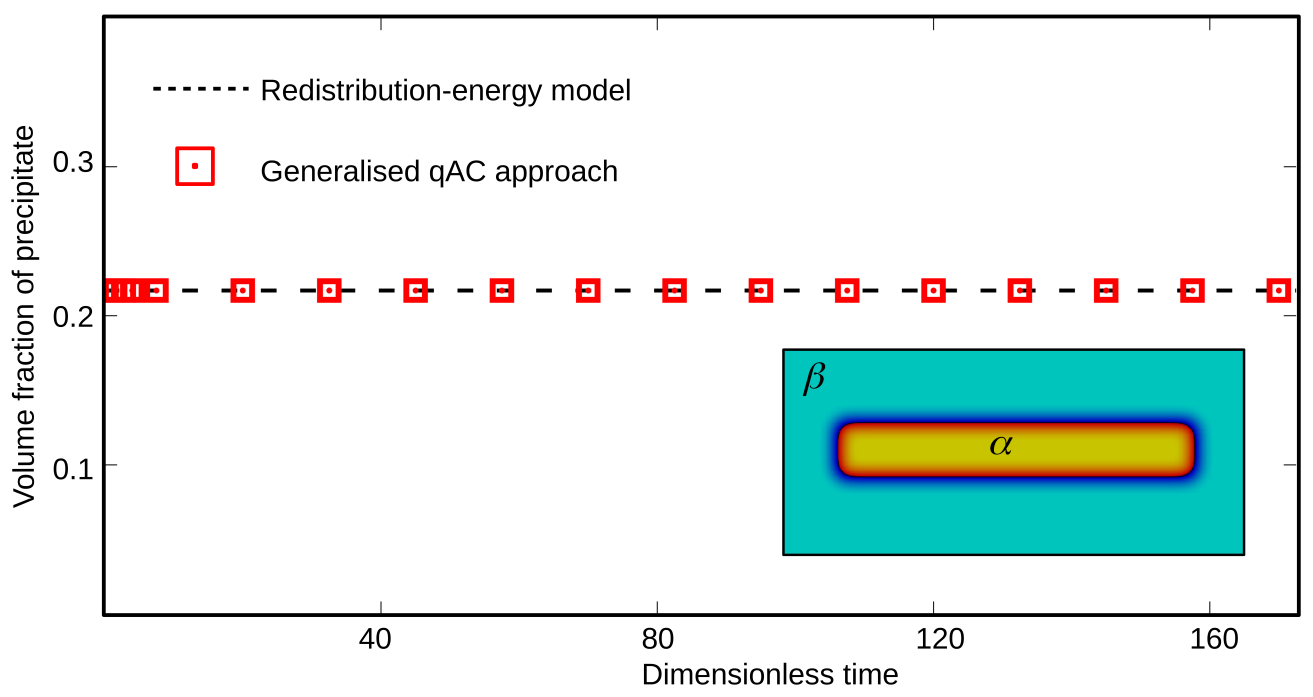

Figure 1: The volume-fraction of the precipitate $-\alpha$ during its morphological evolution governed by the curvaturedifference inherent to the shape. The initial distribution of the phases in the simulation domain is included as a subset of the plot.

generalised quasi-Allen-Cahn (qAC) approach. To that end, a representative microstructure, both in two- and three-dimension, comprising of a precipitate $-\alpha$ in matrix $-\beta$ is considered. The simulation domains devised for the current investigations are homogeneously discretised into voxels of dimension $\Delta \mathrm{x}=\Delta \mathrm{y}=1 \times 10^{-9} \mathrm{~m}$. The evolution equations formulated in the previous sections, Eqns. (14), 21) and (26), are solved over the discretised domain by forward marching Euler's scheme. Approximately, six gridpoints are assigned to the diffuse interface by fixing the length-scale variable at $\varepsilon=3 \Delta \mathrm{x}$. Since the present study primarily focuses on elucidating the limitations of preserving volume in the Allen-Cahn framework, the dimensionless time $t$ is employed for the comparative analyses. Furthermore, the material parameters, including the parabolic fitting for the volume-preserving energy-densities, are adopted from Refs. [48, 50, 44].

\subsection{Volume preserve}

The ribbon-shaped precipitate with rectangular cross-section morphologically evolves into a cylindrical rod governed by the inherent curvature-difference in its shape. This shape-change is referred to as cylinderization [31, 32]. For the two-dimensional investigation, the cross-section of the ribbon-like $\alpha$-precipitate in $\beta$-matrix is considered. The initial configuration of the phases 


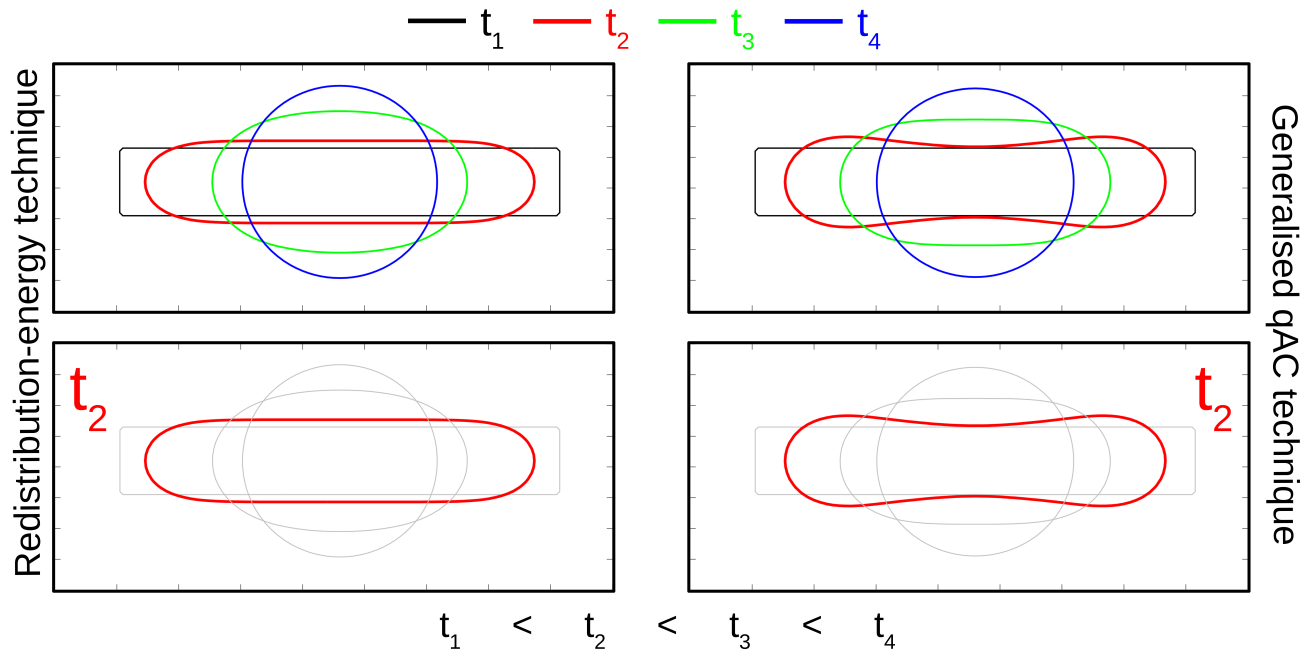

Figure 2: The isoline representation indicating mechanism of cylinderization exhibited by the precipitate in the redistribution-energy and quasi-Allen-Cahn treatment. The temporal change in the morphology of the precipitate under the redistribution-energy model is presented in the left panels, while the outcomes of the qAC model is shown on the right. The precipitate shape at timestep $t_{2}$ is highlighted in red and shown separately.

are illustrated as a subset of Fig. 1. This representative microstructure is allowed to evolve in the redistribution-energy and generalised quasi-Allen-Cahn framework by solving the evolution Eqn. (14) and, Eqns. (21) and (26), respectively.

Through the incremental change in the shape of the precipitate, the system reduces its overall energy-density by minimising the interfacial-energy per unit volume. The volume-fraction of the precipitate during the morphological transformation, under both the approaches compared in the present study, is monitored and presented in Fig. 1 This illustration indicates that the redistribution-energy and generalised quasi-Allen-Cahn technique preserve the volume of the precipitate, despite its temporal evolution. And no visible deviation from the initial phasefraction is observed.

\subsection{Disparity in cylinderization mechanism}

The progressive change in the morphology of the precipitate cross-section, ultimately leading to a disc shape, is shown in Fig. 2 The results rendered by the redistribution-energy and generalised qAC model are collectively depicted in this illustration. Moreover, the contour defined in Eqn. (4) is adopted to cumulatively present the shape-change during the cylinderization. 
Both redistribution-energy and generalised qAC approach invariably allow the cross-section of the precipitate to evolve into a disc-shaped structure. However, cylinderization mechanism adopted by the precipitate vary with the treatment. The difference in the transformation mechanism is visible in shape adopted by the phase- $\alpha$ in timestep $t_{2}$, which is shown separately in Fig. 2

While the shape of the precipitate at timesteps $t_{1}, t_{3}$ and $t_{4}$ are seemingly identical, as shown in Fig. 2. the disparity is evident in timestep $t_{2}$. The redistribution-energy technique preserves the convexity of the phase- $\alpha$ during the evolution, akin to Refs. [33, 37], whereas in the generalised qAC treatment, the convexity in the shape of the precipitate is compromised. In other words, in the redistribution-energy formalism, the curvature-difference is minimised by recession of the termination and a counterbalancing migration of the interface at the mid-section of the precipitate. The local migration of the interface in the central region of the phase- $\alpha$ preserves the volume and renders the characteristic morphology at $t_{2}$, as illustrated in the lower left panel of Fig. 2 The cylinderization mechanism, pertaining to the redistribution-energy framework, can be viewed as morphological transformation wherein the mass from the high-curvature terminations are uniformly deposited in the all flat surfaces. The continual migration of mass from the termination to available flat surfaces transforms the rectangular cross-section to elliptical structural which ultimately assumes a disc shape. In contrast, in the generalised qAC treatment, as shown in right panels of Fig. 2 at $t_{2}$, thick ridges are formed in the longitudinal ends of the precipitate. This longitudinal perturbations indicate that the cylinderization in the generalised qAC model is established by the transfer of mass from the high-curvature source to the immediate-neighbouring sinks (flat surfaces). The flux from the receding termination to the adjacent low-curvature region facilitates the growth of the ridges, which ultimately coalesce to form an elliptical shape that subsequently transforms to a disc.

Experimental observations [31, 32] and theoretical investigations [51, 48, 49] indicate that, of the two fundamentally-different cylinderization mechanisms, illustrated Fig. 2 the outcomes of the generalised qAC technique are physically and thermodynamically consistent than the redistribution-energy model. Therefore, adopting redistribution-energy approach to simulate curvature-driven morphological evolution in two-dimensional microstructure would render an 


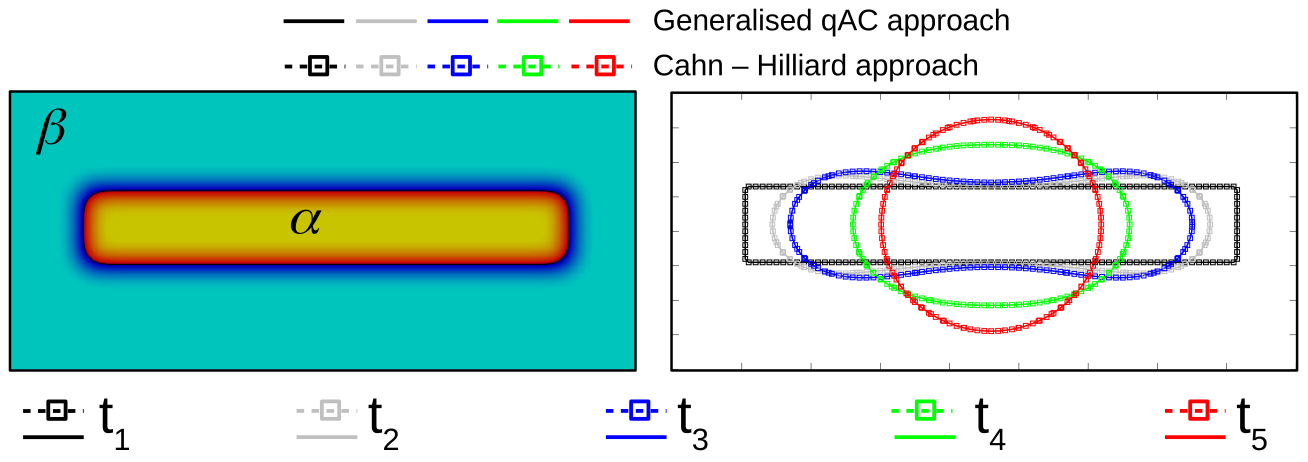

Figure 3: Temporal change in the cross-section of the precipitate, as an isoline representation, accompanying the cylinderization process in the Cahn-Hilliard treatment is overlaid on the outcomes of the generalised quasi-AllenCahn approach.

inaccurate transformation mechanism, eventhough an agreeable final morphology might be established.

\subsection{Consistency of generalised quasi-Allen-Cahn approach}

As introduced earlier, the conventional technique to simulate curvature-driven transformations, wherein the volume-fractions of the phases are preserved, involves treating the phase-field as a conserved variable [39]. Although a variant of the generalised quasi-Allen-Cahn approach, involving concentration as the conserved variable, is employed as an alternate for the CahnHilliard treatment, the outcomes of these two technique have hardly been compared [52, 53, 44]. To expound the thermodynamical consistency of the present generalised qAC formalism, the results are compared with the Cahn-Hilliard approach.

For the current energy-density consideration in Eqn. (16), devoid of any bulk contributions, the phase-field evolution in the Cahn-Hilliard framework is expressed as

$$
\varepsilon \frac{\partial \phi(\vec{x}, t)}{\partial t}=-\nabla \cdot\left[M(\phi(\vec{x}, t)) \nabla\left(2 \varepsilon \gamma \Delta \phi(\vec{x}, t)-\frac{\gamma}{\varepsilon} \frac{16}{\pi^{2}}[1-2 \phi(\vec{x}, t)]\right)\right],
$$

where $M(\phi(\vec{x}, t))$ dictates the mobility of the phase-field [54]. The right panel of Fig. 3 shows the isoline representation of the morphological transformation rendered by this approach for the initial configuration illustrated in the left panel. For comparison, the results of the generalised qAC treatment, formulated in the present work, is overlaid on the outcomes of the Cahn-Hilliard 


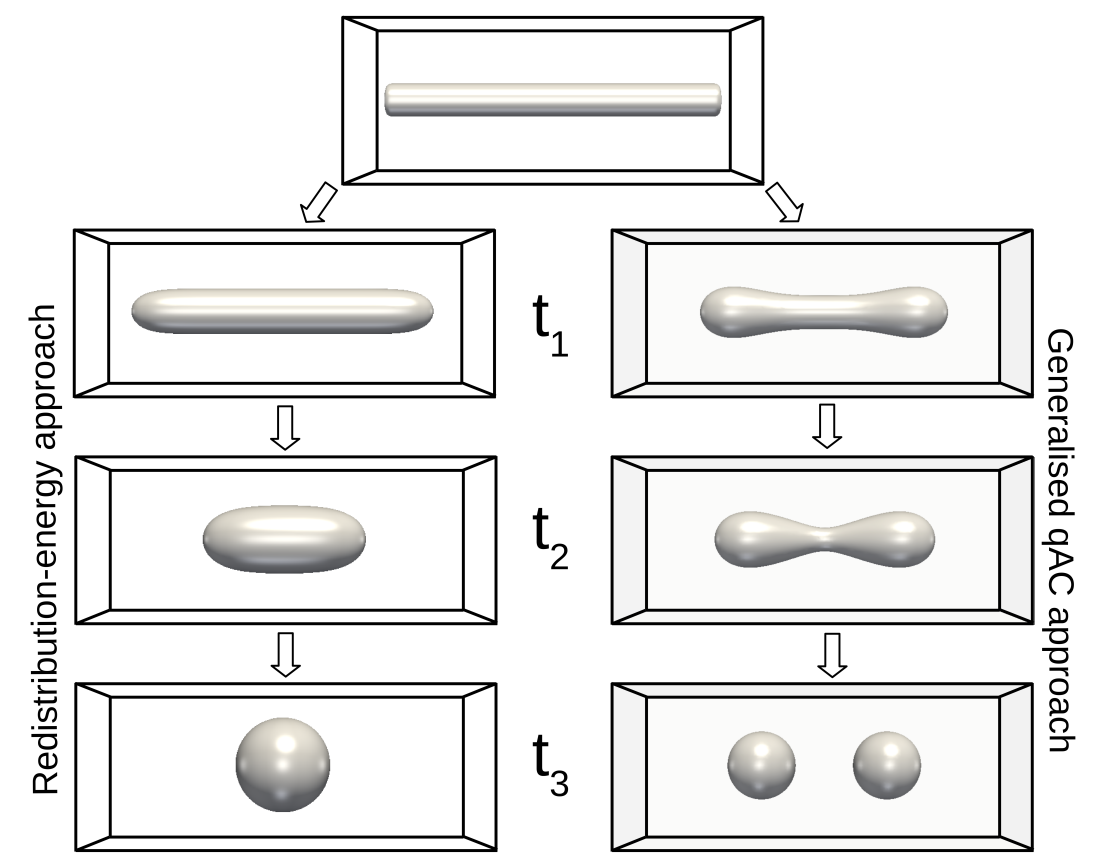

Figure 4: The spheroidisation mechanism exhibited by a three-dimensional uncapped rod in the redistributionenergy framework is compared with the generalised qAC results.

approach. The excellent agreement in the interface profile, at different timesteps, of the two techniques asserts the thermodynamical consistency and the volume-preserving capability of generalised quasi-Allen-Cahn formalism.

\subsection{Comparing three-dimensional spheroidisation}

For the comparative analysis of the outcomes rendered by the redistribution-energy and generalised qAC approach in a three-dimensional setup, the spheroidisation of the uncapped rod is considered. An uncapped rod, owing to the curvature-difference between the termination and remnant flat surface, transforms into a spheroid [55, 56]. The spheroidisation mechanism is fundamentally dictated by the aspect-ratio of the rod, which is the ratio of the length to its width [51, 50].

An uncapped three-dimensional rod of aspect ratio 10, as shown in Fig. 4 is allowed to evolve primarily governed by the inherent curvature-difference in its initial shape, under redistributionenergy and generalised qAC formulations. The outcomes of both these techniques are include in the illustration Fig. 4 
Previous experimental observations [55], analytical [51] and numerical investigations [50] have shown that spheroidisation mechanism of uncapped rod of aspect ratio 10 involves 'ovulation', wherein a single precipitate splits into two, ultimately, forming two spheroids. As shown in Fig. 4, the results of the generalised quasi-Allen-Cahn treatment are consistent with the existing reports. However, the redistribution-energy formalism renders a termination-migration governed direct spheroidisation of the precipitate. This noticeable disparity in the transformation mechanism can be attributed to the failure of the redistribution-energy treatment to capture the appropriate evolution of the interface, which results in the longitudinal perturbation. Moreover, while the ultimate distribution of the phases consists of two spheroids in generalised qAC technique, a single spherical precipitate is formed at the end of the transformation in redistributionenergy technique.

In two-dimensional setup, as discussed in Sec. 3.2 , the redistribution-energy approach establishes the expected final-morphological configuration, while digressing in the transformation mechanism. However, in the three-dimensional consideration, in the addition to the deviation in the spheroidisation mechanism, this technique fails to achieve thermodynamically-consistent and analytically-predicted final distribution of the phases.

\subsection{Capturing thermodynamic nature of system}

A numerical technique can be deemed 'quantitative' only when its outcomes reflect the thermodynamical nature of the system. For instance, in a domain comprising of two closed systems with different-sized elliptical precipitate associated with each, as shown in Fig. 5, a quantitative treatment renders an evolution wherein the morphological change in one precipitate is independent of the other. In other words, in a domain wherein the mass transfer across the precipitate is thwarted by a barrier, the Ostwald ripening should not be introduced despite the differences in the size of phase- $\alpha$.

To examine the quantitative nature of the redistribution and generalised quasi-Allen-Cahn approach, a setup as illustrated in Fig. 5 is considered. As opposed to previous domain configuration, in this setup zero-Neumann condition is imposed along the boundaries. The barrier, along with the appropriate boundary condition, prevents the flux across the domain. The temporal 


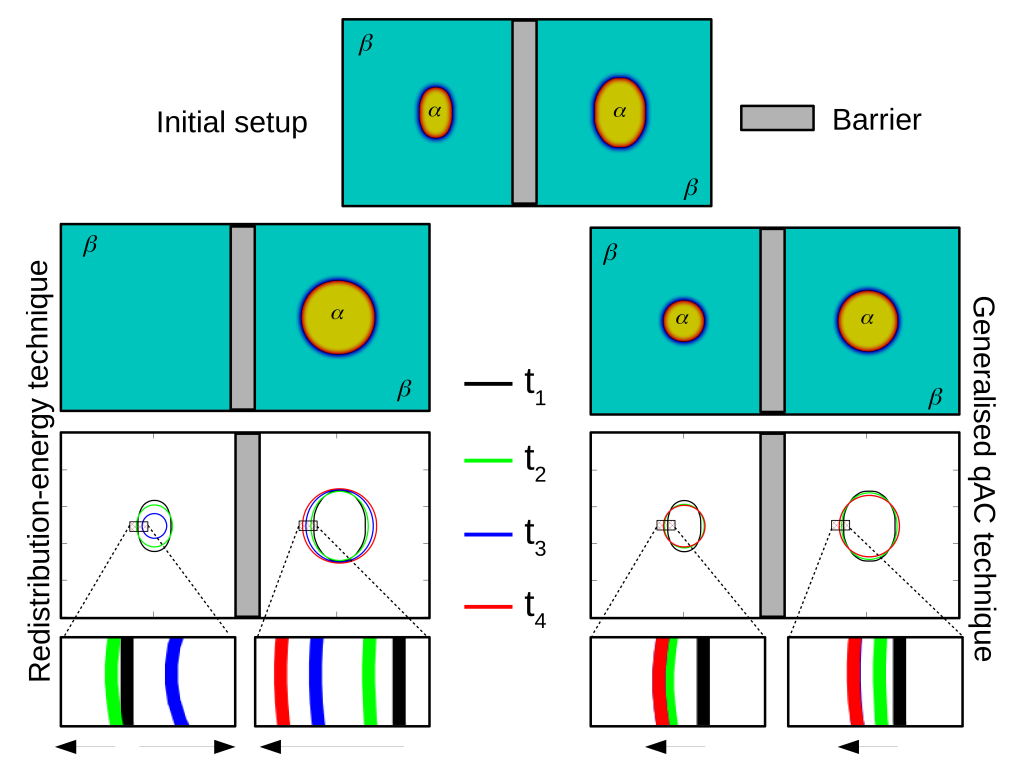

Figure 5: The morphological evolution of the precipitates in a domain comprising of two closed-system in the redistribution-energy and generalised quasi-Allen-Cahn framework.

evolution of the phases under the redistribution-energy and generalised quasi-Allen-Cahn technique is shown in Fig. 5 In the generalised qAC treatment, the transformation complies with the imposed thermodynamical condition, and the precipitates transform independently into a disc shape. Whereas, inspite of distinguishing the domain into two closed-systems by introducing a barrier, a morphological change which reflects coarsening through Ostwald ripening is rendered by the redistribution-energy technique. This evolution of a representative setup in Fig. 5 indicates that the redistribution-energy formalism is not entirely quantitative.

\section{Conclusion}

Owing to its versatility, and proven thermodynamical-consistency, phase-field modelling have been extensively adopted to investigate microstructural evolutions. In addition to the phase transformations, the microstructural changes include morphological transformation of the phases in chemical equilibrium, which is characterised by temporally-invariable phase-fractions. In the phase-field framework, curvature-driven transformations, wherein the volume of the phases are preserved, are conventionally treated by Cahn-Hilliard approach. This approach preserves the 
volume by treating the phase-field as a conserved variable. However, owing to the computational and numerical intricacies associated with the Cahn-Hilliard treatment, other alternatives are formulated to render volume-preserved morphological evolution governed by curvature. The limitations of one such alternate, which hitherto has not been considered, is elucidated in this work by comparative analysis.

As opposed to the phase-field techniques wherein the volume of the evolving phase is preserved by the inclusion of an appropriate Lagrange multiplier, the redistribution-energy treatment conserves the phase-fraction through bulk contribution. Despite this unique formulation, the present study shows that the morphological evolution, particularly the preservation of convexity, rendered by the redistribution-energy approach is similar to the Lagrange-multiplier based techniques. Moreover, when compared to the generalised quasi-Allen-Cahn treatment,

which is realised to be consistent with the well-established Cahn-Hilliard approach, the outcomes to be redistribution-energy technique are inaccurate, and deviate noticeably from the experimental observations and theoretical predictions. The limitations of employing the redistributionenergy approach to investigate microstructural transformations can be summarised as:

- In two-dimensional condition, the redistribution-energy technique establishes an agreeable final microstructural configuration. However, it is identified that the transformation mechanism, which renders this phase distribution, is physically improbable and thermodynamically incoherent.

- The inability of the redistribution-energy approach to recover the appropriate mechanism adversely effects the evolution in three-dimension. The microstructure emerging from the redistribution-energy treatment is fundamentally different from the existing analytical and numerical reports.

- In its current formulation, the phase-field evolution under the redistribution-energy technique do not reflect the thermodynamical condition imposed on the system.

Given the aforementioned limitations of preserving volume in Allen-Cahn framework, immense attention should be paid before adopting this approach for microstructural investigations. More- 
over, an extensive comparative study focusing on the numerical efficiency of the quasi-AllenCahn technique when compared to the Cahn-Hilliard will be reported in the upcoming works.

\section{Acknowledgments}

PG Kubendran Amos thanks the financial support of the German Research Foundation (DFG) under the project AN 1245/1. E Schoof and D Schneider contributed to the discussions and the results through the initiative 'Virtmat' and the programme-EMR of the Helmholtz association. Fruitful discussions with Paul Hoffrogge is acknowledged.

\section{References}

\section{References}

[1] John Wilson Martin, John Wilson Martin, Roger Davidge Doherty, and B Cantor. Stability of microstructure in metallic systems. Cambridge University Press, 1997.

[2] Robert E Reed-Hill and Reza Abbaschian. Physical metallurgy principles. 1973.

[3] Harry Bhadeshia and Robert Honeycombe. Steels: microstructure and properties. Butterworth-Heinemann, 2017.

[4] Salvatore Torquato. Random heterogeneous materials: microstructure and macroscopic properties, volume 16. Springer Science \& Business Media, 2013.

[5] MP Puls and JS Kirkaldy. The pearlite reaction. Metallurgical and Materials Transactions B, 3(11):2777-2796, 1972.

[6] William W Mullins and Robert F Sekerka. Morphological stability of a particle growing by diffusion or heat flow. Journal of applied physics, 34(2):323-329, 1963.

[7] FA Nichols and WW Mullins. Morphological changes of a surface of revolution due to capillarity-induced surface diffusion. Journal of Applied Physics, 36(6):1826-1835, 1965.

[8] Plapp Mathis. Phase-field modelling of solidification microstructures. Journal of the Indian Institute of Science, 96(3):179-198, 2016.

[9] Mathis Plapp. Phase-field models. In Handbook of Crystal Growth, pages 631-668. Elsevier, 2015.

[10] Long-Qing Chen. Phase-field models for microstructure evolution. Annual review of materials research, 32(1):113-140, 2002.

[11] Nikolas Provatas and Ken Elder. Phase-field methods in materials science and engineering. John Wiley \& Sons, 2011. 
[12] Robert F Almgren. Second-order phase field asymptotics for unequal conductivities. SIAM fournal on Applied Mathematics, 59(6):2086-2107, 1999.

[13] LT Mushongera, PG Kubendran Amos, B Nestler, and K Ankit. Phase-field simulations of pearlitic divergence in fe-c-mn steels. Acta Materialia, 150:78-87, 2018.

[14] PG Kubendran Amos, Ephraim Schoof, Nick Streichan, Daniel Schneider, and Britta Nestler. Phase-field analysis of quenching and partitioning in a polycrystalline fe-c system under constrained-carbon equilibrium condition. Computational Materials Science, 159:281-296, 2019.

[15] Seong Gyoon Kim, Won Tae Kim, and Toshio Suzuki. Phase-field model for binary alloys. Physical review e, 60(6):7186, 1999.

[16] J Eiken, B Böttger, and I Steinbach. Multiphase-field approach for multicomponent alloys with extrapolation scheme for numerical application. Physical review E, 73(6):066122, 2006.

[17] PG Kubendran Amos, Ephraim Schoof, Daniel Schneider, and Britta Nestler. Chemo-elastic phase-field simulation of the cooperative growth of mutually-accommodating widmanstätten plates. Fournal of Alloys and Compounds, 767:1141-1154, 2018.

[18] Jingzhi Zhu, Long-Qing Chen, Jie Shen, and Veena Tikare. Coarsening kinetics from a variable-mobility cahnhilliard equation: Application of a semi-implicit fourier spectral method. Physical Review E, 60(4):3564, 1999.

[19] CE Krill Iii and L-Q Chen. Computer simulation of 3-d grain growth using a phase-field model. Acta materialia, 50(12):3059-3075, 2002.

[20] Britta Nestler, Harald Garcke, and Björn Stinner. Multicomponent alloy solidification: phase-field modeling and simulations. Physical Review E, 71(4):041609, 2005.

[21] I Steinbach and F Pezzolla. A generalized field method for multiphase transformations using interface fields. Physica D: Nonlinear Phenomena, 134(4):385-393, 1999.

[22] Samuel M Allen and John W Cahn. A microscopic theory for antiphase boundary motion and its application to antiphase domain coarsening. Acta metallurgica, 27(6):1085-1095, 1979.

[23] Jingtang Ma, Yingjun Jiang, and Kaili Xiang. Numerical simulation of blowup in nonlocal reaction-diffusion equations using a moving mesh method. Journal of Computational and Applied Mathematics, 230(1):8-21, 2009.

[24] Xiaofeng Ren and Juncheng Wei. On a phase field problem driven by interface area and interface curvature. European Journal of Applied Mathematics, 20(6):531-556, 2009.

[25] Doug Stafford, Michael J Ward, and Brian Wetton. The dynamics of drops and attached interfaces for the constrained allen-cahn equation. European fournal of Applied Mathematics, 12(1):1-24, 2001.

[26] Yoshihiro Suwa, Yoshiyuki Saito, and Hidehiro Onodera. Phase field simulation of grain growth in three dimensional system containing finely dispersed second-phase particles. Scripta Materialia, 55(4):407-410, 2006.

[27] Nele Moelans, Bart Blanpain, and Patrick Wollants. Quantitative analysis of grain boundary properties in a generalized phase field model for grain growth in anisotropic systems. Physical Review B, 78(2):024113, 2008. 
[28] Ramanathan Perumal, PG Kubendran Amos, Michael Selzer, and Britta Nestler. Phase-field study on the formation of first-neighbour topological clusters during the isotropic grain growth. Computational Materials Science, 140:209-223, 2017.

[29] Ramanathan Perumal, PG Kubendran Amos, Michael Selzer, and Britta Nestler. Phase-field study of the transient phenomena induced by 'abnormally'large grains during 2-dimensional isotropic grain growth. Computational Materials Science, 147:227-237, 2018.

[30] HE Cline. Shape instabilities of eutectic composites at elevated temperatures. Acta Metallurgica, 19(6):481-490, 1971.

[31] TH Courtney and JC Malzahn Kampe. Shape instabilities of plate-like structures-ii. analysis. Acta Metallurgica, 37(7):1747-1758, 1989.

[32] JC Malzahn Kampe, Thomas H Courtney, and Yang Leng. Shape instabilities of plate-like structures-i. experimental observations in heavily cold worked in situ composites. Acta Metallurgica, 37(7):1735-1745, 1989.

[33] Jacob Rubinstein and Peter Sternberg. Nonlocal reaction-diffusion equations and nucleation. IMA fournal of Applied Mathematics, 48(3):249-264, 1992.

[34] Guy Barles, H Mete Soner, and Panagiotis E Souganidis. Front propagation and phase field theory. SIAM fournal on Control and Optimization, 31(2):439-469, 1993.

[35] Guillermo Sapiro, Allen Tannenbaum, et al. Area and length preserving geometric invariant scale-spaces. 1993.

[36] Junseok Kim, Seunggyu Lee, and Yongho Choi. A conservative allen-cahn equation with a space-time dependent lagrange multiplier. International fournal of Engineering Science, 84:11-17, 2014.

[37] Morgan Brassel and Elie Bretin. A modified phase field approximation for mean curvature flow with conservation of the volume. Mathematical Methods in the Applied Sciences, 10(34):1157-1180, 2011.

[38] Dongsun Lee and Junseok Kim. Comparison study of the conservative allen-cahn and the cahn-hilliard equations. Mathematics and Computers in Simulation, 119:35-56, 2016.

[39] John W Cahn and John E Hilliard. Free energy of a nonuniform system. i. interfacial free energy. The fournal of chemical physics, 28(2):258-267, 1958.

[40] Martin Heida, Josef Málek, and Kumbakonam R Rajagopal. On the development and generalizations of cahnhilliard equations within a thermodynamic framework. Zeitschrift für angewandte Mathematik und Physik, 63(1):145-169, 2012.

[41] Harald Garcke, Britta Nestler, Björn Stinner, and Frank Wendler. Allen-cahn systems with volume constraints. Mathematical Models and Methods in Applied Sciences, 18(08):1347-1381, 2008.

[42] Britta Nestler, Frank Wendler, Michael Selzer, Björn Stinner, and Harald Garcke. Phase-field model for multiphase systems with preserved volume fractions. Physical Review E, 78(1):011604, 2008.

[43] Oleg Tschukin, Alexander Silberzahn, Michael Selzer, Prince GK Amos, Daniel Schneider, and Britta Nestler. Concepts of modeling surface energy anisotropy in phase-field approaches. Geothermal Energy, 5(1):19, 2017. 
[44] PG Kubendran Amos, Ephraim Schoof, Daniel Schneider, and Britta Nestler. On the globularization of the shapes associated with alpha-precipitate of two phase titanium alloys: Insights from phase-field simulations. Acta Materialia, 159:51-64, 2018.

[45] PG Kubendran Amos, Avisor Bhattacharya, Britta Nestler, and Kumar Ankit. Mechanisms of pearlite spheroidization: Insights from 3d phase-field simulations. Acta Materialia, 161:400-411, 2018.

[46] PG Kubendran Amos, Ephraim Schoof, Daniel Schneider, and Britta Nestler. On the volume-diffusion governed termination-migration assisted globularization in two-phase solid-state systems: Insights from phase-field simulations. In Numerical Modelling in Engineering, pages 47-63. Springer, 2018.

[47] Tobias Mittnacht, PG Kubendran Amos, Daniel Schneider, and Britta Nestler. Understanding the influence of neighbours on the spheroidization of finite 3-dimensional rods in a lamellar arrangement: Insights from phase-field simulations. In Numerical Modelling in Engineering, pages 290-299. Springer, 2018.

[48] PG Kubendran Amos, LT Mushongera, and Britta Nestler. Phase-field analysis of volume-diffusion controlled shape-instabilities in metallic systems-i: 2-dimensional plate-like structures. Computational Materials Science, 144:363-373, 2018.

[49] Prince Gideon Kubendran Amos. Understanding the volume-diffusion governed shape-instabilities in metallic systems. PhD thesis, Karlsruher Institut für Technologie (KIT), 2019.

[50] PG Kubendran Amos, LT Mushongera, Tobias Mittnacht, and Britta Nestler. Phase-field analysis of volumediffusion controlled shape-instabilities in metallic systems-ii: Finite 3-dimensional rods. Computational Materials Science, 144:374-385, 2018.

[51] FA Nichols. On the spheroidization of rod-shaped particles of finite length. fournal of materials science, 11(6):1077-1082, 1976.

[52] Hamed Ravash, Jef Vleugels, and Nele Moelans. Three-dimensional phase-field simulation of microstructural evolution in three-phase materials with different diffusivities. Fournal of materials science, 49(20):7066-7072, 2014.

[53] Vishal Yadav, Liesbeth Vanherpe, and Nele Moelans. Effect of volume fractions on microstructure evolution in isotropic volume-conserved two-phase alloys: A phase-field study. Computational Materials Science, 125:297$308,2016$.

[54] Jay Santoki, Arnab Mukherjee, Daniel Schneider, Michael Selzer, and Britta Nestler. Phase-field study of electromigration-induced shape evolution of a transgranular finger-like slit. Fournal of Electronic Materials, 48(1):182-193, 2019.

[55] S Marich. Structural stability of the rod-like iron-iron sulfide eutectic at elevated temperatures. Metallurgical Transactions, 1(10):2953-2958, 1970.

[56] M McLean. The kinetics of spheroidization of lead inclusions in aluminium. Philosophical Magazine, 27(6):12531266, 1973. 